\title{
Diagnosis of carotid artery stenosis with oculopneumoplethysmography alone and in combination with MRA
}

This article was published in the following Dove Press journal:

Vascular Health and Risk Management

22 November 2012

Number of times this article has been viewed

\section{Richard M Elias' \\ John T Wald ${ }^{2}$ \\ David F Kallmes ${ }^{2}$}

'Department of Internal Medicine, ${ }^{2}$ Department of Radiology, Mayo Clinic, Rochester, MN, USA
Correspondence: David F Kallmes Department of Radiology, Mayo Clinic, 200 First Street SW, Rochester, MN 55905, USA

$\mathrm{Tel}+\mathrm{I} 5072644348$

Fax + I 5072664372

Email kallmes.david@mayo.edu
Background: The purpose of this study was to assess the accuracy of oculopneumoplethysmography (OPG) for the diagnosis of carotid artery stenosis both alone and in conjunction with carotid magnetic resonance angiography (MRA).

Methods: This retrospective study reviewed patients who had undergone both OPG and digital subtraction angiography (DSA, 90 patients, 174 vessels) within two weeks to determine the accuracy of OPG with DSA as the reference standard for the detection of carotid artery stenosis. Three carotid artery stenosis thresholds ( $\geq 50 \%, \geq 70 \%, \geq 80 \%$ ) were analyzed. The accuracy of the combination of OPG and MRA was analyzed in a subset of patients who underwent OPG and MRA and DSA (53 patients, 94 vessels).

Results: The sensitivity and negative predictive value of OPG increased with higher-degree carotid artery stenoses, and for lesions $\geq 80 \%$, these values were $85.3 \%$ and $94.2 \%$, respectively. Specificity and positive predictive values were lower at all thresholds, and were $72.9 \%$ and $49.3 \%$, respectively, at the $\geq 80 \%$ stenosis threshold. When OPG and MRA were concordant, the sensitivity and specificity for carotid artery stenoses $\geq 80 \%$ were $91.0 \%$ and $97.8 \%$, respectively. OPG correctly identified $71.4 \%$ of false-positive and $80.0 \%$ of false-negative magnetic resonance angiographies for that degree of carotid artery stenosis.

Conclusion: OPG appears to be an accurate rule-out test for hemodynamically significant carotid artery stenosis. OPG augments the accuracy of MRA for detection of carotid artery disease.

Keywords: oculopneumoplethysmography, carotid artery stenosis, magnetic resonance angiography, diagnosis, stroke

\section{Introduction}

Stroke results in more than 140,000 deaths in the US per year, making it the third leading cause of mortality behind heart disease and cancer. ${ }^{1}$ The proportion of stroke attributable to carotid atherosclerosis has proved difficult to quantify, but hemodynamically significant carotid artery stenosis has been known to be associated with increased incidence of stroke for half a century. ${ }^{2}$ The principal goal of investigating carotid artery stenosis is to identify the group of patients for whom carotid disease warrants surgical or percutaneous intervention; however, the modalities currently in general use to assess the carotid system are associated with various limitations. Currently, three noninvasive modalities are in common use to assess the carotid arteries, ie, carotid duplex ultrasound (CDUS), computed tomographic angiography, and magnetic resonance angiography (MRA). Each of these modalities has disadvantages. CDUS is known to be significantly operator-dependent, to be limited by a variety of factors such as patient body habitus and vascular anatomic variation, and to overestimate stenoses. MRA is 
time-consuming, requires a paramagnetic contrast agent for higher quality images, and is limited by patient factors, such as claustrophobia or metal implants. Computed tomographic angiography requires a bolus of iodinated contrast and exposes the patient to ionizing radiation. All are expensive. Oculopneumoplethysmography (OPG) is a fast, relatively inexpensive, noninvasive investigation, initially conceived in the 1970s, which uses measurement of ocular systolic pressures in order to make determinations regarding the presence of hemodynamically significant carotid artery stenoses.

William Gee, a surgeon at the National Naval Medical Center and later The Allentown Hospital, did much of the work on the underlying physiology and development of the OPG device..$^{3-6}$ Given the tremendous variability in human body weight and brain size, the adult human eye is of surprisingly uniform size. The normal intraocular pressure is 10-25 millimeters of mercury (mmHg), which is to a large extent maintained by oculovascular fluid dynamics combined with a minimal element of sclera elasticity. Closing the eye raises the pressure to $50 \mathrm{mmHg}$. Rubbing the eye elevates the pressure to the order of $150-250 \mathrm{mmHg} .^{5}$ The fundamental physical property utilized by OPG is that outward distortion of the sclera increases tension without a change in volume, resulting in elevated intraocular pressure. Consequently, there is a delineated relationship between the degree of vacuum applied to the sclera and the resulting elevation of intraocular pressure. Elevation to a level greater than the ophthalmic systolic pressure results in obliteration of the ocular pulsation. ${ }^{5}$

The process of OPG involves attaching suction cups equipped to measure the blood pressure waveform in the eye to the sclera bilaterally. Vacuum pressure is applied to the sclera sufficient to occlude ophthalmic arterial flow and is then incrementally reduced until systolic flow returns, which is the ocular systolic pressure (OSP) in a process analogous to the use of a sphygmomanometer to measure brachial systolic pressure (BSP). The ophthalmic artery is a distal segment of the internal carotid artery, but the collateral flow provided by the circle of Willis necessitates measurement of the pressure in both ophthalmic arteries simultaneously in the assessment of potential internal carotid artery stenosis, otherwise the location of a putative causative lesion is ambiguous.

The technique for brachial and OPG measurement is described in the Methods section. A BSP:OSP coordinate is plotted on a graph. The mean value for OSP for any given BSP is calculable from the formula OSP $=55+0.43$ BSP $^{6}$ This formula was derived from the study of normal human subjects and from baboon studies using direct measurement of intraocular pressure and carotid artery pressure via posterior chamber and carotid artery needle cannulation. ${ }^{4}$ The plotting of OSP for the range of BSPs creates an oblique line on a graph with BSP on the $\mathrm{x}$ axis and OSP on the $\mathrm{y}$ axis. The standard error of the estimates associated with this line is $8 \mathrm{mmHg}$. The "line of demarcation" which delineates the lower limit of a normal OPG study is 2 standard deviations below the mean of normal, giving a threshold of OSP $=39+0.43$ BSP. The test is considered positive for carotid stenosis if one or both OSPs fall below the line of demarcation or if one OSP is lower than the contralateral OSP by a magnitude of $5 \mathrm{mmHg}$ or greater. ${ }^{6}$ For the reader with an interest in greater detail regarding the physiological underpinning and computational elements of OPG, please see the comprehensive reviews by Gee and Chen., ${ }^{3,7}$

OPG was in quite widespread use during the 1980s and into the early 1990s, having been in regular practice in greater than 300 centers in the US. ${ }^{8}$ It was one of the principal investigations for carotid artery stenosis but fell largely to the wayside as CDUS imaging of the carotid circulation came to supplant it. Even though OPG is not widely used at present, there are reasons to consider its relevance carefully. The currently used imaging techniques suffer relative challenges in accuracy, and may require ionizing radiation, iodinated contrast, costly imaging equipment, or entail risk to the patient in the form of atheroembolic disease. Furthermore, imaging techniques rely on morphologic features or evidence for disturbed flow patterns to detect stenosis. OPG is unique among noninvasive techniques for carotid artery stenosis detection in that it relies on finding deficient pressure in the end organ, thereby providing direct insight into cerebrovascular hemodynamics. As such, OPG might be complementary to morphologically based noninvasive imaging techniques for carotid artery stenosis detection, such as MRA. Previous work has indeed shown the use of OPG to improve the accuracy of CDUS. ${ }^{9}$ The purpose of this study was to revisit this modality and critically assess its accuracy and applicability to modern carotid artery stenosis assessment. A secondary goal was to determine the value of OPG in combination with MRA for the accurate detection of hemodynamically significant carotid artery stenosis. Of particular interest is whether as an adjunct to MRA, OPG might reduce the need for invasive angiography in determining which patients to refer for carotid surgery.

We have undertaken a retrospective review of patients who underwent both OPG and digital subtraction angiography (DSA) in short succession in order to determine the degree of correlation between OPG and the gold standard and whether OPG is an investigation of clinical utility in the diagnosis of 
carotid artery stenosis. There was some minimal variation in the DSA technique, but it generally involved placement of a 5 French sheath in a common femoral artery using a Seldinger technique. In some cases, arch aortogram was performed via a 5 French pigtail catheter placed in the ascending aorta prior to catheterization of the common carotid arteries. In all cases, a catheter (generally 5 French) was used to catheterize the left and right common carotid arteries selectively for cervical and cerebral biplane, digital subtraction angiography. Typical iodinated contrast injections were aortic arch $30 \mathrm{~mL}(15 \mathrm{~mL} / \mathrm{sec})$, common carotid artery $8 \mathrm{~mL}(6 \mathrm{~mL} / \mathrm{sec})$, and internal carotid $6 \mathrm{~mL}(4 \mathrm{~mL} / \mathrm{sec})$. The DSA gold standard for measurement of carotid stenosis was the NASCET (North American Symptomatic Carotid Endarterectomy Trial) criteria.

We further examined a subset of patients who underwent DSA, OPG, and carotid system MRA to determine the degree of concordance between OPG and MRA, the accuracy of these two modalities in combination for diagnosing clinically significant carotid artery stenosis, and whether OPG could be used to improve the accuracy of MRA.

\section{Materials and methods}

This research was approved by the Mayo Clinic Institutional Review Board (ID 08-006494). The physiologic aspects of OPG are described briefly above and in greater detail elsewhere. ${ }^{3-6}$ All OPG studies were undertaken on a OPG$\mathrm{Gee}^{\circledR}$ ocular pneumoplethysmograph (Electro-Diagnostic
Instruments, Burbank CA). Each study takes approximately 15 minutes to perform. Manual blood pressure is taken in both arms and the higher systolic value used as the comparator with the OPG. With the patient in a supine position, anesthetic eye drops are applied bilaterally. Eyecups with a $14 \mathrm{~mm}$ outside diameter are applied to the sclera lateral to the cornea and a partial vacuum is initiated by a foot switch. The remainder of the vacuum sequence is automated by the instrument. The instrument has three transducers and a three-channel recorder. Two of the transducers sense the return of ocular pulsations as the vacuum falls below the systolic pressure in the respective ophthalmic arteries. The third transducer measures in continuous fashion the magnitude of vacuum being applied to both sclera. Two channels record ocular pressure waveforms while the third channel is an electrocardiogram which ensures that ocular pulsations are not artifactual by coordination with the cardiac cycle (see Figures 1 and 2 for normal and abnormal ocular pulsation tracings, respectively.) Recording is obtained during continuous decrement of the applied vacuum. The eyecups are in place for approximately 30 seconds. All OPG studies are interpreted by a neurologist assigned to the neurovascular laboratory. See Figures 3 and 4 for reports of normal and abnormal OPGs, respectively, which correspond with the tracings in Figures 1 and 2.

We reviewed the medical records at Mayo Clinic, Rochester, for all patients who underwent both OPG and DSA in close proximity between 1999 and 2008. All patients

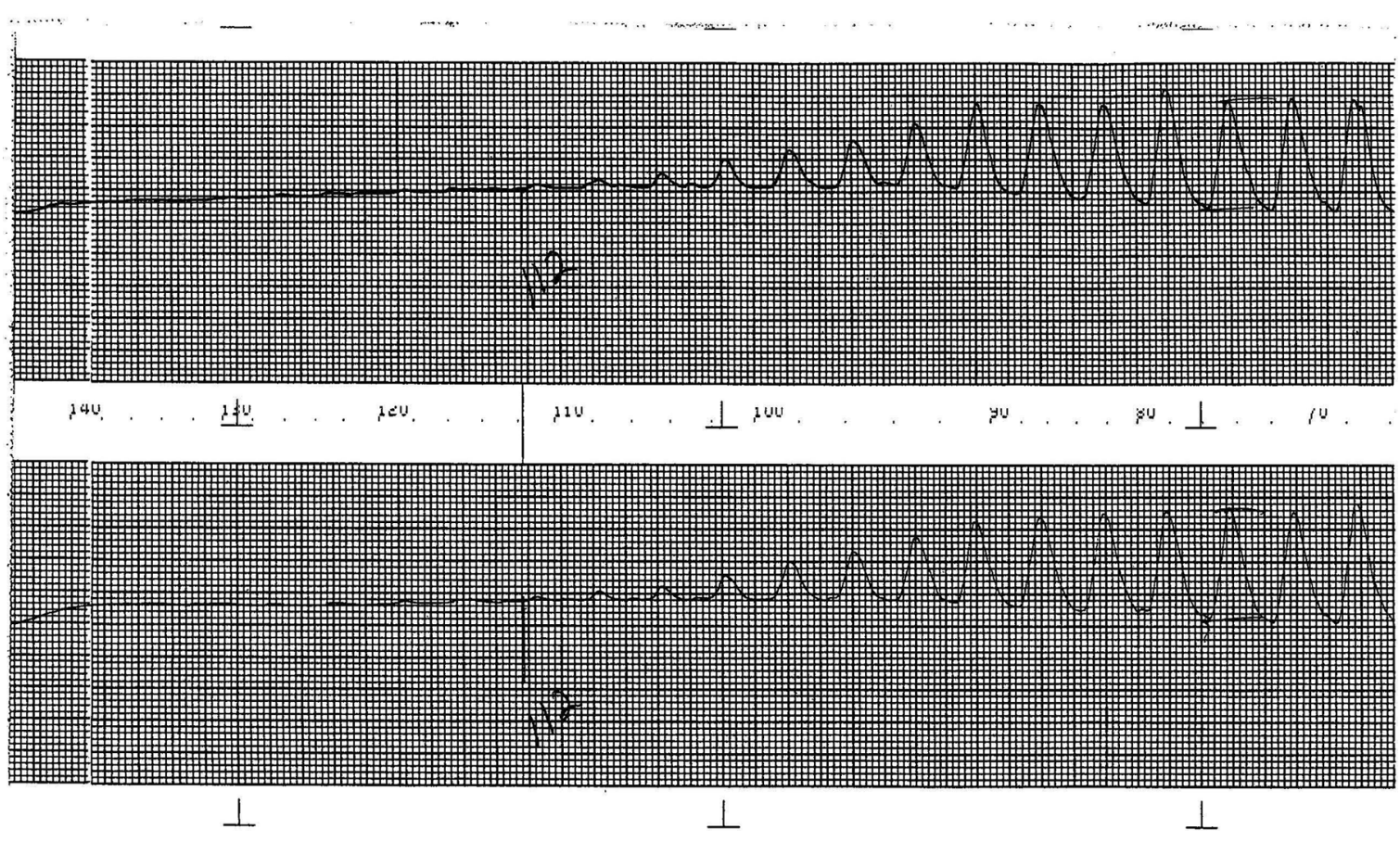

Figure I An oculopneumoplethysmography tracing demonstrating a normal study. The return of ocular pulsations occurs at I/ $2 \mathrm{mmHg}$ bilaterally. See Figure 3 for the report of this study. 


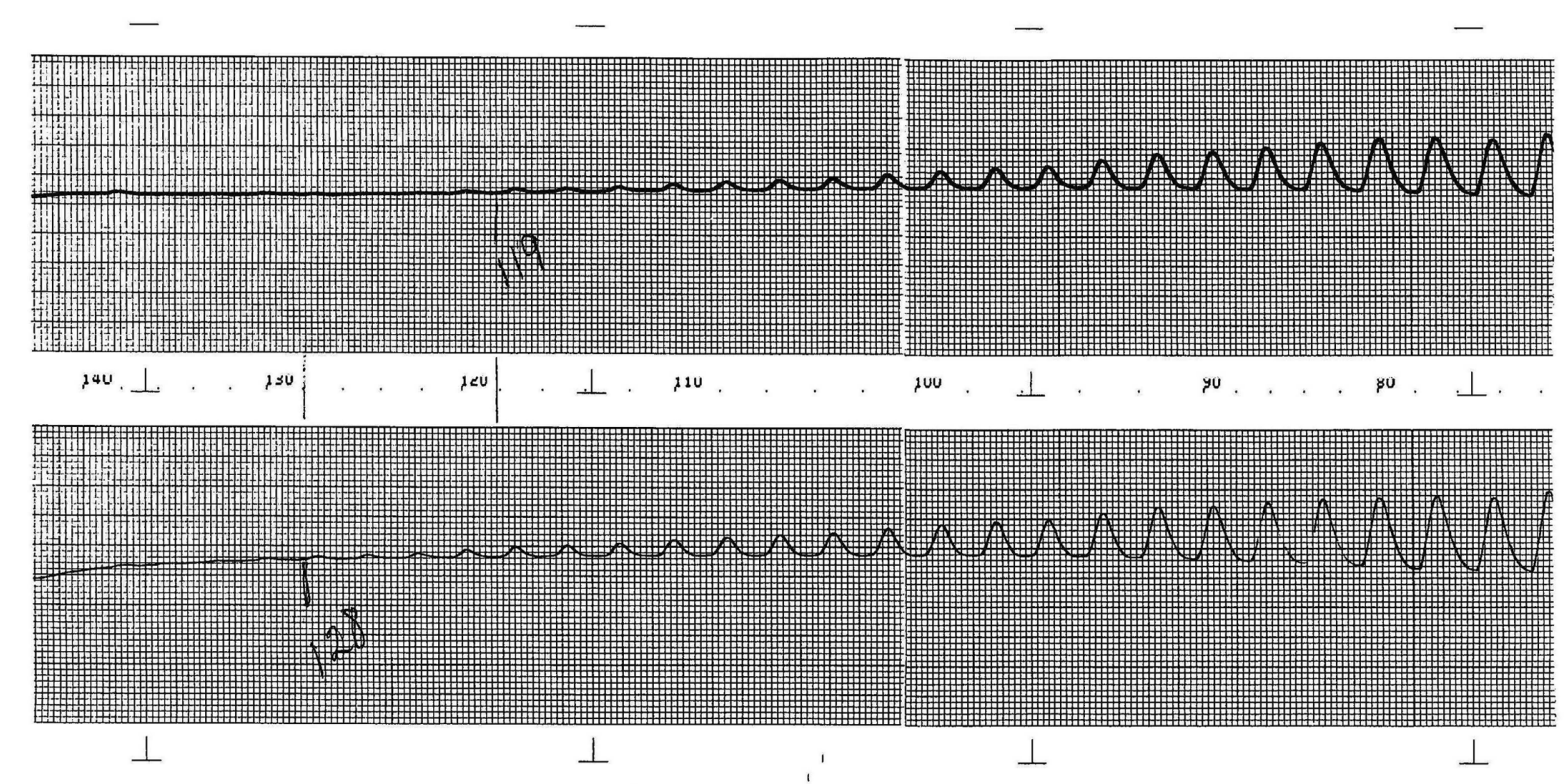

Figure 2 An oculopneumoplethysmography tracing demonstrating a pressure significant lesion on the right side. The return of ocular pulsations occurs at I28 mmHg on the left but not until $119 \mathrm{mmHg}$ on the right. See Figure 4 for the report of this study.

referred to the vascular laboratory for OPG who had DSA within two weeks of the study were eligible. The need for OPG and DSA was at the discretion of the referring physician. The review identified 100 patients (192 vessels) who had undergone both OPG and carotid angiography consecutively within a two-week period between 1999 and 2008. Eight patients were excluded due to interval endarterectomy between angiography and OPG. Two patients were excluded due to failure to locate their OPG report. This resulted in 90 patients (174 vessels) for inclusion. A subset of 53 patients who had undergone OPG, DSA, and carotid system MRA over a one-month period was identified. In an attempt to standardize MRA interpretation and ensure that a percentage carotid stenosis was recorded, all MRAs were independently reviewed by an experienced neuroradiologist (JTW) blinded to the results of both the OPG and DSA.

Thresholds for stenoses were set at $50 \%, 70 \%$, and $80 \%$ by DSA. Accuracy of OPG for identifying stenoses at these three cut-off levels was determined. Additionally, accuracy of MRA alone and of OPG in conjunction with MRA for diagnosis of carotid artery stenosis at these same thresholds was undertaken in the subset of patients who underwent all three studies.

\section{Results}

When compared with DSA, OPG demonstrated sensitivity, specificity, a positive predictive value (PPV), a negative predictive value (NPV), and positive and negative likelihood ratios (LR+/LR-) as detailed in Table 1. Of note from this data is that, for lesions $\geq 70 \%$, the sensitivity was $78.0 \%$, which rose to $85.4 \%$ for lesions $\geq 80 \%$. NPVs at the $70 \%$ and $80 \%$ levels of stenoses were $87.4 \%$ and $94.2 \%$, respectively. The LR- in this group with higher-grade lesions was 0.171 . There was a significant number of false-positive results, particularly if the threshold for stenosis was set high, reflected in PPVs of 77.5\%, $64.8 \%$, and $49.3 \%$ at the $\geq 50 \%, \geq 70 \%$ and $\geq 80 \%$ lesion thresholds, respectively. Compared with unilateral lesions, sensitivity for bilateral stenoses was inferior for lesions of $\geq 50 \%$ and $\geq 70 \%$ but was $100 \%$ for lesions $\geq 80 \%$, although the absolute number of the latter was small $(n=4$, Table 1$)$.

Although not the primary focus of this study, the performance of MRA alone is presented in Table 2. Of note is that while specificity and the NPV were high $(92.3 \%$ and $92.1 \%$ at the $70 \%$ and $80 \%$ stenosis thresholds), the PPV was only moderate (63.2\% for the highest grade group), suggesting a significant number of false-positive results. Of interest was the use of OPG and MRA in conjunction. The results of this analysis are detailed in Table 3. When OPG and MRA are in agreement for both positive and negative studies, the performance of this combination is very accurate. Sensitivity and the PPV were both $91.0 \%$ for $\geq 80 \%$ stenoses, with specificity and the NPV of $97.8 \%$. When MRA was inaccurate for higher grade lesions, OPG was able to identify false-positive and false negative studies correctly in $71.4 \%$ and $80.0 \%$ of cases, respectively (Table 4). 


\section{Neurovascular examination \\ Clinical neurovascular laboratory Mayo Clinic - Rochester, Minnesota Requested by,}

Referral diagnosis: amaurosis fugax

\begin{tabular}{|l|l|l|}
\hline Study & Right & Left \\
\hline Oculoplethysmography (OPG) & & \\
\hline Supine & $112 \mathrm{mmHg}$ & $112 \mathrm{mmHg}$ \\
\hline Arterial doppler & & \\
\hline Periorbital & Normal & Normal \\
\hline Vertebral & & \\
\hline Brachial systolic pressures & & \\
\hline Supine & $140 \mathrm{mmHg}$ & $142 \mathrm{mmHg}$ \\
\hline
\end{tabular}

\section{Observations}

Right

Left

Comments: Normal studies.

\section{Preliminary}

Performed

Printed

Page 1

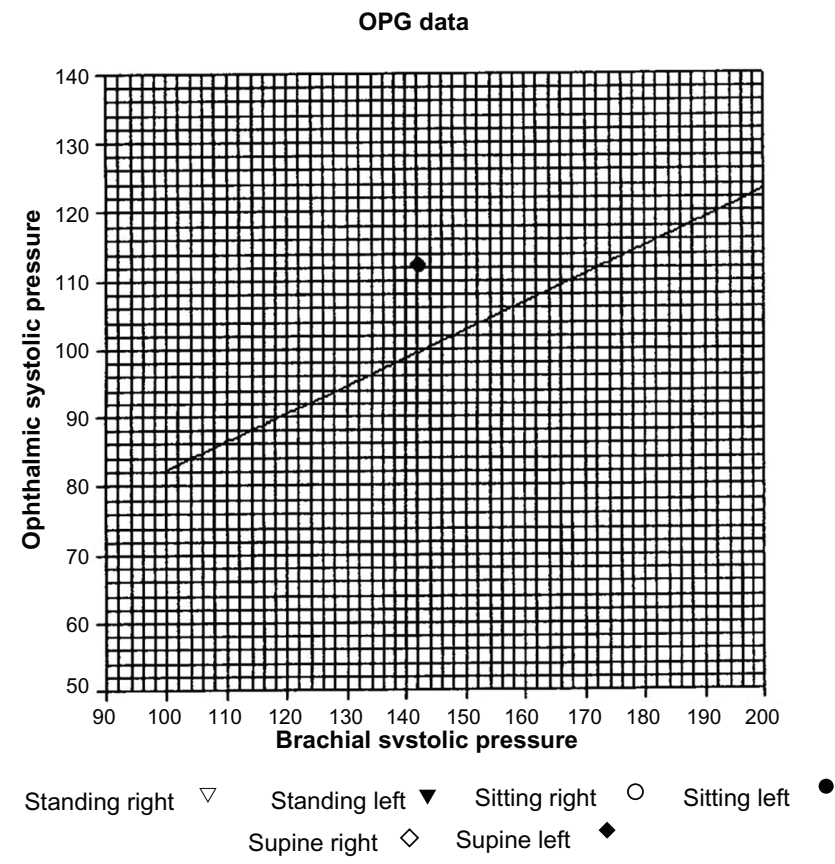

Technician

Consultant

***End of report ${ }^{* * *}$

Figure 3 Report of the normal study represented in Figure I. Both ophthalmic systolic pressures lie above the line of demarcation on the graph (two data points are superimposed one on another) and are within $5 \mathrm{mmHg}$ one to the other.

\section{Discussion}

For lesions generally regarded as hemodynamically significant and appropriate for intervention, the accuracy of OPG appears comparable with other available tests, most notably CDUS. ${ }^{10}$ Because OPG is a physiological test of diminished carotid blood flow, the data demonstrate that it lacks accuracy for detecting stenoses of $50 \%-69 \%$; which is not surprising because these lesions are generally not flow-limiting. There is little evidence to suggest that detection of such lesions is of benefit for most patients. Viewed in that context, a "false-negative" result for a low grade stenosis on a binary (yes/no) examination such as OPG is potentially viewed as a success, because it correctly identified the lesion as not flow-limiting and likely not to benefit from intervention, particularly if in an asymptomatic patient.

The high NPV and LR - for categories of lesions greater than $70 \%$ and $80 \%$ suggest that, as an initial test among patients referred due to clinical suspicion for carotid disease, OPG is accurate at identifying those in whom no further testing is required. Of note, all vessels for which the examinations 


\section{Neurovascular examination \\ Clinical neurovascular laboratory \\ Sex: F Mayo Clinic - Rochester, Minnesota \\ Requested by,}

Referral diagnosis: transient ischmic attack (TIA)

\begin{tabular}{|l|l|l|}
\hline Study & Right & Left \\
\hline Oculoplethysmography (OPG) & & \\
\hline Supine & $119 \mathrm{mmHg}$ & $128 \mathrm{mmHg}$ \\
\hline Arterial doppler & & \\
\hline Periorbital & Normal & Normal \\
\hline Vertebral & & \\
\hline Brachial systolic pressures & & \\
\hline Supine & $168 \mathrm{mmHg}$ & $174 \mathrm{mmHg}$ \\
\hline
\end{tabular}

Observations

Right

Left

Comments: The OPG is consistent with the presence of a right internal carotid artery system pressure significant lesion. Adverse change on the right since exam of 3/26/2003.
Preliminary

Performed

Printed

Page 1

2 previous exam(s)

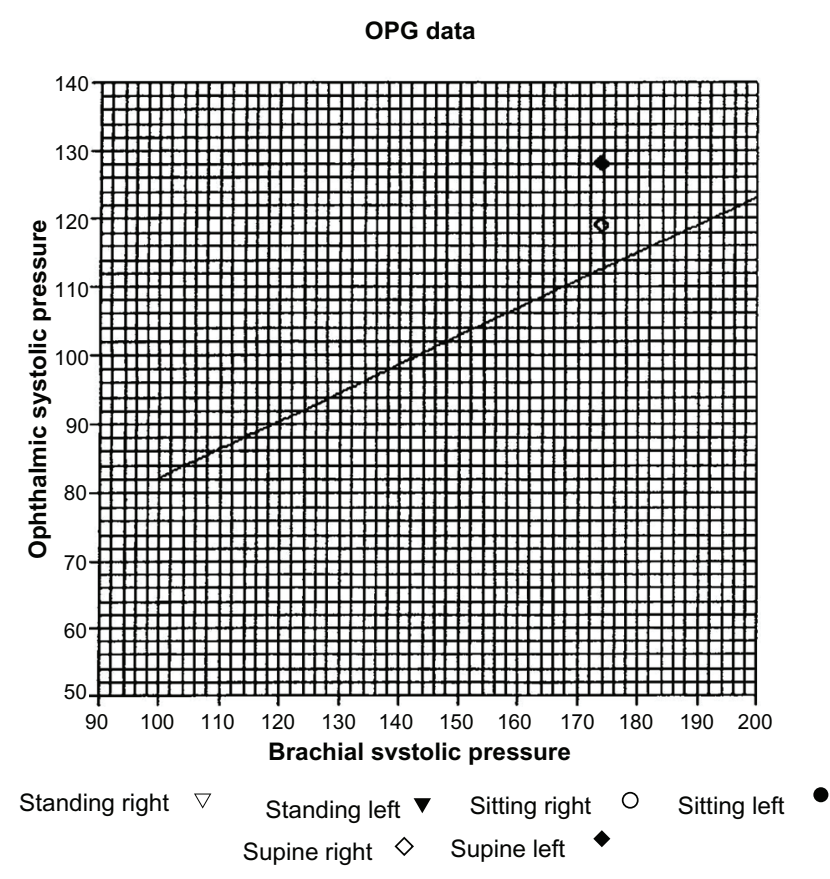

Technician

Consultant

***End of report***

Figure 4 Report of the abnormal study represented in Figure 2. Both data points are above the line of demarcation but the right side pressure is 9 mmHg less than left, consistent with a pressure significant lesion involving the right carotid system.

Table I Oculopneumoplethysmography results with digital subtraction angiography as the reference standard

\begin{tabular}{|c|c|c|c|c|c|c|}
\hline & Sensitivity (\%) & Specificity (\%) & PPV (\%) & NPV (\%) & LR+ & LR- \\
\hline $50 \%$ stenosis $(\mathrm{n}=92)$ & 57.3 & 79.5 & 77.5 & 60.2 & 2.77 & 0.535 \\
\hline $70 \%$ stenosis $(n=59)$ & 78.0 & 78.3 & 64.8 & 87.4 & 3.59 & 0.281 \\
\hline $80 \%$ stenosis $(n=40)$ & 85.4 & 72.9 & 49.3 & 94.2 & 3.23 & 0.171 \\
\hline \multicolumn{7}{|l|}{ Bilateral stenoses } \\
\hline $50 \%$ & $51(n=40)$ & & & & & \\
\hline $70 \%$ & $57(n=14)$ & & & & & \\
\hline $80 \%$ & $100(n=4)$ & & & & & \\
\hline
\end{tabular}

Abbreviations: PPV, positive predictive value; NPV, negative predictive value; LR, likelihood ratio. 
Table 2 Magnetic resonance angiography results with digital subtraction angiography as the reference standard

\begin{tabular}{|c|c|c|c|c|c|c|}
\hline & Sensitivity (\%) & Specificity (\%) & PPV (\%) & NPV (\%) & LR+ & LR- \\
\hline $50 \%$ stenosis $(n=42)$ & 76.2 & 77.5 & 78.1 & 75.6 & 2.77 & 0.535 \\
\hline $70 \%$ stenosis $(n=22)$ & 81.8 & 82.8 & 64.3 & 92.3 & 4.67 & 0.221 \\
\hline $80 \%$ stenosis $(n=17)$ & 70.6 & 89.2 & 63.2 & 92.1 & 6.54 & 0.330 \\
\hline
\end{tabular}

Abbreviations: PPV, positive predictive value; NPV, negative predictive value; LR, likelihood ratio.

were undertaken were included without reference to the vessel for which the clinical suspicion was focused. This has the potential to inflate the specificity and NPV because it includes vessels for which there was little pretest suspicion. These data suggest that OPG may not be sufficiently specific, nor have adequate PPV to be the sole basis for making a determination regarding carotid intervention. The results demonstrate that performing endarterectomy on the strength of a positive OPG alone would likely result in a significant amount of unnecessary surgery ( $14 \%$ of arteries if a $70 \%$ stenosis threshold were used). In the subgroup analysis of patients with bilateral lesions, OPG appeared to be less sensitive than for unilateral lesions, although the sample size was small. This is not surprising given that a difference between right and left ophthalmic artery pressures is one of the two fundamental OPG criteria for designating an artery as stenotic, but does represent a potential limitation of the test.

The false-positive rate for MRA was relatively low, but sensitivity was less than has previously been reported at only $71 \%$ for stenoses greater than $80 \% .{ }^{10}$ When OPG was combined with MRA, confirmation of a positive MRA was highly specific and an accurate predictor of disease. Similarly, when OPG and MRA are concordant for the absence of disease, the performance approaches $100 \%$. This suggests that if OPG confirms MRA findings of no significant disease, these patients need no further investigation. If OPG confirms a diseased vessel detected by MRA, the likelihood that this represents true disease is high. OPG correctly identified $71 \%$ of false-positive vessels on MRA and $80 \%$ of false negatives. OPG is a potential adjunct to MRA, and when discordant, the likelihood that MRA is inaccurate should be considered.

There are some factors other than carotid artery stenosis which potentially influence OPG measurement. There is an element of operator dependence in discerning the return of ocular pulsations from artifact, necessitating adequate training prior to use of the device. The inability to measure brachial systolic pressure accurately due to body habitus or upper limb trauma or surgery is another potential source of inaccuracy although this has not been noted to be a significant barrier to OPG at our institution. It has been previously shown that operation and anesthesia can affect the fluid dynamics of the eye, making short-term postoperative studies potentially unreliable. ${ }^{6}$ Diseases affecting the cranial vasculature, such as giant cell arteritis, moyamoya vasculopathy, and carotidcavernous sinus fistula, have also been shown to influence OPG measurements. ${ }^{11-13}$

The results described here are similar to previously undertaken studies of OPG; however, caution is warranted for several reasons. Most of these studies were undertaken in the 1970s and 1980s, so gold standard assessments for carotid stenoses were often based on criteria which predate NASCET, and therefore tend to overestimate stenoses by utilizing normal carotid proximal to the lesion as the reference for stenosis. ${ }^{14}$ Further, previous studies compared OPG with cerebral angiography in an era prior to the availability of digital subtraction. Finally, the criteria for assessing an OPG result has significantly evolved over time to reflect technical improvements to the machines, and in the "heyday" of OPG there was no uniformity of criteria between centers, making direct comparisons between studies problematic. ${ }^{8}$ The above notwithstanding, our findings are generally in line with those of other investigators and our own institution's experience with this modality. ${ }^{15-17}$

OPG appears to be safe and well tolerated. The time during which the suction cups are applied to the sclera is approximately 30 seconds to one minute. The 28 years of experience at our institution has demonstrated very uncommon

Table 3 Accuracy of concordant oculopneumoplethysmography and magnetic resonance angiography

\begin{tabular}{|c|c|c|c|c|c|c|}
\hline & Sensitivity (\%) & Specificity (\%) & PPV (\%) & NPV (\%) & LR+ & LR- \\
\hline $50 \%$ stenosis $(n=18)$ & 77.8 & 86.7 & 77.8 & 86.7 & 5.85 & 0.256 \\
\hline $70 \%$ stenosis $(n=13)$ & 100.0 & 93.3 & 81.2 & 100.0 & 14.92 & 0.00 \\
\hline $80 \%$ stenosis $(\mathrm{n}=\mathrm{II})$ & 91.0 & 97.8 & 91.0 & 97.8 & 41.36 & 0.092 \\
\hline
\end{tabular}

Abbreviations: PPV, positive predictive value; NPV, negative predictive value; LR, likelihood ratio. 
Table 4 Oculopneumoplethysmography accuracy on vessels with inaccurate magnetic resonance angiography

\begin{tabular}{lll}
\hline & $\begin{array}{l}\text { MRA false } \\
\text { positive }\end{array}$ & $\begin{array}{l}\text { MRA false } \\
\text { negative }\end{array}$ \\
\hline $50 \%$ stenosis (\% OPG correct) & $55.6 \%(n=9)$ & $40.0 \%(n=10)$ \\
$70 \%$ stenosis (\% OPG correct) & $60.0 \%(n=10)$ & $50.0 \%(n=4)$ \\
$80 \%$ stenosis (\% OPG correct) & $71.4 \%(n=7)$ & $80.0 \%(n=5)$ \\
\hline
\end{tabular}

Abbreviations: OPG, oculopneumoplethysmography; MRA, magnetic resonance angiography.

occurrences of scleral hemorrhage and rare corneal abrasion, without any permanent or vision-affecting events. A case series has demonstrated no morbidity associated with OPG in the setting of previous lens implantation, although a small series has suggested that OPG may be inaccurate in the setting of glaucoma. ${ }^{18,19}$ Although no specific evidence exists, previous history of retinal detachment has been taken as a relative contraindication to the procedure, although it has been performed in this circumstance at our institution without adverse outcome.

The study data covers a 10 -year period. A long time span was necessary to accrue a sufficient number of cases, given that OPG is performed infrequently at our institution. The benefit of the low volume of OPGs is that all studies were performed by one of two technicians on identical equipment, reducing bias and error over the 10-year time span. Regarding MRA, there have been some technological improvements over the time period, but none that are felt to significantly change the accuracy of determining carotid artery stenosis by gadolinium bolus imaging. Given that a number of radiologists had interpreted the MRAs over the ten-year period, all of these studies were reread by a neuroradiologist (JTW) blinded to the DSA and OPG results to reduce the likelihood of interpreter bias.

CDUS is currently the prevailing modality for initial assessment and ongoing monitoring of the carotid system. The limitations of carotid ultrasound, including a high falsepositive rate and difficulties of interpretation associated with transposed, hyperdynamic, previously intervened on vessels and patient body habitus are well described. ${ }^{20,21}$ Furthermore, as the ophthalmic is an end-artery of the carotid, OPG assesses pressure effects present throughout the entire anterior circulation whilst CDUS is limited to evaluation of the cervical portion of the carotid artery. OPG is also advantageous in that it is an objective process less limited by the operator dependence known in CDUS. ${ }^{22}$ Further, because choroid blood flow, which makes up the bulk of ocular blood flow, is not autoregulated, it enables detection of hemodynamically significant lesions in a manner more sensitive than autoregulated cerebral blood flow assessable via transcranial Doppler ultrasound..$^{23}$ However, a limitation is that OPG does not provide information regarding the morphologic characteristics of carotid plaques, which may have prognostic implications regarding likelihood of stroke, although this remains unproven..$^{24,25}$

In the current climate of health care costs vastly outstripping economic growth and imaging services on the leading edge of this cost explosion, the notion of an inexpensive investigation which might better select patients for whom expensive imaging is likely to be beneficial is attractive. The cost of bilateral assessment of the carotid circulation by OPG is $\$ 469$ at our institution and the scarcity of materials and replacement parts for the device suggest that if it were in widespread use the cost would be lower. While not without limitations, it appears that OPG may have a role in the diagnosis of carotid artery stenosis that warrants further investigation. Of benefit moving forward would be a randomized prospective trial assessing OPG compared with and in conjunction with modern CDUS. Furthermore, given the increasing prevalence of cerebrovascular disease, research examining the potential utility and cost-effectiveness of OPG as a screening tool for asymptomatic high-risk patients would also be of significance. OPG appears to be a test with good efficacy for the noninvasive assessment of carotid artery stenosis both alone and particularly in conjunction with MRA.

\section{Acknowledgment}

The authors would like to thank Anna Elias for her invaluable assistance in the preparation of this manuscript.

\section{Disclosure}

The authors have no conflicts of interest to disclose.

\section{References}

1. American Heart Association. Heart Disease and Stroke Statistics, 2010. Available from: http://www.americanheart.org/downloadable/ heart/1265665152970DS-3241\%20HeartStrokeUpdate_2010.pdf.

2. Fisher M. Occlusion of the internal carotid artery. AMA Arch Neurol Psychiatry. 1951;65:346-377.

3. Gee W, Smith CA, Hinson CE, Wylie EJ. Ocular pneumoplethysmography in carotid artery disease. Med Instrum. 1974;8:244-248.

4. Gee W, Oller DW, Wylie EJ. Noninvasive diagnosis of carotid occlusion by ocular pneumoplethysmography. Stroke. 1976;7:18-21.

5. Gee W. Ocular pneumoplethysmography. Surv Ophthalmol. 1985;29: 276-292.

6. Gee W. Carotid physiology with ocular pneumoplethysmography. Stroke. 1982;13:666-673.

7. Chen C, Reed JF 3rd, Rice DC, Gee W, Updike DP, Salathe EP. Biomechanics of ocular pneumoplethysmography. J Biomech Eng. 1993;115:231-238.

8. Eikelboom B, Riles TS, Folcarelli P, Imparato AM. Criteria for interpretation of ocular pneumoplethysmography (Gee). Arch Surg. 1983;118:1169-1172. 
9. Castaldo JE, Nicholas GG, Gee W, Reed JF. Duplex ultrasound and ocular pneumoplethysmography concordance in detecting severe carotid stenosis. Arch Neurol. 1989;46:518-522.

10. Nederkoorn PJ, Mali WP, Eikelboom BC, et al. Preoperative diagnosis of carotid artery stenosis: accuracy of noninvasive testing. Stroke. 2002;33:2003-2008

11. Bosley TM, Savino PJ, Sergott RC, Eagle RC, Sandy R, Gee W. Ocular pneumoplethysmography can help in the diagnosis of giant-cell arteritis. Arch Ophthalmol. 1989;107:379-381.

12. Muppal M, Castaldo JE. Unilateral supraclinoid internal carotid artery stenosis with moyamoya-like vasculopathy. Noninvasive assessments. J Neuroimaging. 1994;4:11-16.

13. Gee W, Morrow RA, Stephens HW, Lin FZ. Ocular pneumoplethysmography in carotid-cavernous sinus fistulas. J Neurosurg. 1984;59: 40-45.

14. Rothwell PM, Gibson RJ, Slattery J, Sellar RJ, Warlow CP. Equivalence of measurements of carotid stenosis. A comparison of three methods on 1001 angiograms. European Carotid Surgery Trialists' Collaborative Group. Stroke. 1994;25:2435-2439.

15. Schwartz JA, Flanigan P, Schuler JJ, Ryan TJ, Castronuovo JJ. Indirect assessment of carotid occlusive disease by ocular pneumoplethysmography. $500 \mathrm{mmHg}$ vacuum pressure measurements and ocular pulse timing. Stroke. 1984;15:521-526.

16. Wiebers DO, Folger WN, Forbes GS, Younge BR, O'Fallon WM. Ophthalmodynamometry and ocular pneumoplethysmography for detection of carotid occlusive disease. Arch Neurol. 1982;39:690-691.
17. Baker JD, Barker WF, Machleder HI. Evaluation of extracranial cerebrovascular disease with ocular pneumoplethysmography. Am J Surg. 1978;136:206-208.

18. Gee W. Ocular pneumoplethysmography after lens implantation. $J$ Cataract Refract Surg. 1988;14:417-420.

19. Shakin EP, Bosley TM, Gee W, et al. Abnormal ocular pneumoplethysmographic results in unilateral neovascular glaucoma. Arch Neurol. 1989;46:1308-1310.

20. Qureshi AI, Suri MF, Ali Z, et al. Role of conventional angiography in evaluation of patients with carotid artery stenosis demonstrated by Doppler ultrasound in general practice. Stroke. 2001;32:2287-2291.

21. Johnston DC, Goldstein LB. Clinical carotid endarterectomy decision making: noninvasive vascular imaging versus angiography. Neurology. 2001;56:1009-1015.

22. Criswell BK, Langsfeld M, Tullis MJ, Marek J. Evaluating institutional variability of duplex scanning in the detection of carotid artery stenosis. Am J Surg. 1998;176:591-597.

23. Gee W, Perline RK, Madden AE. Physiology of carotid endarterectomy with ocular pneumoplethysmography. J Vasc Surg. 1986;4:129-135.

24. Takaya N, Yuan C, Chu B, et al. Association between carotid plaque characteristics and subsequent ischemic cerebrovascular events: a prospective assessment with MRI - initial results. Stroke. 2006;37: 818-823.

25. Hatsukami TS, Ferguson MS, Beach KW, et al. Carotid plaque morphology and clinical events. Stroke. 1997;28:95-100.
Vascular Health and Risk Management

\section{Publish your work in this journal}

Vascular Health and Risk Management is an international, peerreviewed journal of therapeutics and risk management, focusing on concise rapid reporting of clinical studies on the processes involved in the maintenance of vascular health; the monitoring, prevention and treatment of vascular disease and its sequelae; and the involvement of

\section{Dovepress}

metabolic disorders, particularly diabetes. This journal is indexed on PubMed Central and MedLine. The manuscript management system is completely online and includes a very quick and fair peer-review system, which is all easy to use. Visit http://www.dovepress.com/ testimonials.php to read real quotes from published authors. 